\title{
Special issue on developing nature-inspired intelligence by neural systems
}

\author{
Carlos M. Travieso-González ${ }^{1}$ Jesús B. Alonso-Hernández ${ }^{1}$
}

Published online: 8 November 2020

(C) Springer-Verlag London Ltd., part of Springer Nature 2020

Many specifics of neural systems are not well addressed by the conventional models currently used in different applications. The purpose of the special issue is to present and discuss novel ideas, work and results related to alternative techniques of neural system approaches based on bio-inspired intelligence, which depart from mainstream procedures.

Recent studies based on complex system opened new doors in research field and in particular improved the quality and the results of diverse applications. Neural system approaches on bio-inspired intelligence contributed a lot to this fact, also in areas like speech, biomedicine, biometrics, signals and images, and other applications.

This special issue aims to bring together researchers to report the latest research findings and developments of bioinspired intelligence and to explore future research directions.

This special issue includes works in three areas, which are set in the following topics;

- Study of disease with nature-inspired intelligence

- Analysis of signals with nature-inspired intelligence

- Applications on nature-inspired intelligence

The area "Study of disease with nature-inspired intelligence" includes three works. The first work proposes a self-configuring classification combination method for the detection of the obstructive sleep apnea [1]. The work is applied on different datasets and, automatically, is able to choose the most discriminate features and classifier

Carlos M. Travieso-González

carlos.travieso@ulpgc.es

Jesús B. Alonso-Hernández

jesus.alonso@ulpgc.es

1 Institute for Technological Development and Innovation in Communications, Signals and Communications Department, IDeTIC-ULPGC, University of Las Palmas de Gran Canaria, Las Palmas de Gran Canaria, Spain adjustment. Besides, in [2], an approach is developed for the identification of cardiac diseases by phonocardiogram signals. This work applies an extraction of discriminatory features, which are classified by machine learning approaches. The accuracy was $97.78 \%$ with an equal error rate of $2.22 \%$ for abnormal and normal heart sound classification. Finally, there is a work, which presents a stride detection for patient with Parkinson's disease and control persons [3]. It is implemented by convolutional neural networks, dynamic time warping and algorithms min-max patterns. It reached a F1-score of 0.938 , a recall of 0.968 and a precision of 0.910 .

For the area "Analysis of speech with nature-inspired intelligence," there are two works. The first work shows a system for the multilingual audio information management [4], which is based on a scalable architecture of automatic machine learning methodologies for semantic knowledge analysis in complex environments. The accuracies were between 81 and $68 \%$ according to the language. The following paper implements a system for detecting the stressed from non-stressed speech [5], applied a ICA/PCA approach and so, to detect both classes with a support vector machine (SVM) binary. The accuracy was between 86.6 and $83.3 \%$ according to type of SVM kernel.

Finally, the area "Applications on nature-inspired intelligence" is composed by 5 works. In [6], a detection system of text is implemented by the use of deep learning approaches; it is a sequential combination with a convolutional neural network and a long short-term memory approaches. The authors have used Flickr8k and Flickr30k benchmark datasets, reaching an area under the ROC curve of $80.6 \%$ and $79.8 \%$, respectively [6]. Another work presents an approach based on gated multiple ridge descent mechanism for the localization of 3D facial landmark [7]. The localization errors are between 2 and $4.8 \%$ for different public datasets.

The following paper describes an approach based on convolutional neural networks for the xylem segmentation in stained cross-sectional vessels images [8]. This work 
shows different quality measure with accuracies between 72 and $92 \%$ [8]. There is another paper, which shows a new dataset of sclera biometrics for a public use [9]. This dataset has been checked for different applications as the effects of age and gender, variability of gaze direction and image resolution. Its evaluation reached equal error rates between 0.11 and $0.33 \%$. After experiments, convolutional neural network approach is the best method for this identification. In the last paper, the authors include a push-pull layer for $\mathrm{CNN}$ architectures in order to get a better result due to the reduction in the error corruption in the propagation of the network [10]. It was checked with a public dataset, and the validation of this approach shows a reduction in the classification error.

\section{References}

1. Mostafa SS, Mendonça F, Juliá-Serdá G et al (2019) SC3: selfconfiguring classifier combination for obstructive sleep apnea. Neural Comput Appl. https://doi.org/10.1007/s00521-019-045822

2. Yadav A, Singh A, Dutta MK et al (2019) Machine learningbased classification of cardiac diseases from PCG recorded heart sounds. Neural Comput Appl. https://doi.org/10.1007/s00521019-04547-5

3. Steinmetzer T, Bönninger I, Reckhardt M et al (2019) Comparison of algorithms and classifiers for stride detection using wearables. Neural Comput Appl. https://doi.org/10.1007/s00521019-04384-6

4. Lopez-de-Ipina K, Barroso N, Calvo PM et al (2020) Multilingual audio information management system based on semantic knowledge in complex environments. Neural Comput Appl. https://doi.org/10.1007/s00521-019-04618-7

5. Palacios D, Rodellar V, Lázaro C et al (2019) An ICA-based method for stress classification from voice samples. Neural Comput Appl. https://doi.org/10.1007/s00521-019-04549-3

6. Gupta N, Jalal AS (2019) Integration of textual cues for finegrained image captioning using deep CNN and LSTM. Neural Comput Appl. https://doi.org/10.1007/s00521-019-04515-z

7. Križaj J, Peer P, Struc V et al (2019) Simultaneous multi-descent regression and feature learning for facial landmarking in depth images. Neural Comput Appl. https://doi.org/10.1007/s00521019-04529-7

8. Garcia-Pedrero A, García-Cervigón AI, Olano JM et al (2019) Convolutional neural networks for segmenting xylem vessels in stained cross-sectional images. Neural Comput Appl. https://doi. org/10.1007/s00521-019-04546-6

9. Vitek M, Rot P, Štruc V et al (2020) A comprehensive investigation into sclera biometrics: a novel dataset and performance study. Neural Comput Appl. https://doi.org/10.1007/s00521-02004782-1

10. Strisciuglio N, Lopez-Antequera M, Petkov N (2020) Enhanced robustness of convolutional networks with a push-pull inhibition layer. Neural Comput Appl. https://doi.org/10.1007/s00521-02004751-8

Publisher's Note Springer Nature remains neutral with regard to jurisdictional claims in published maps and institutional affiliations. 